\title{
Migratory Logistic Regression for Learning Concept Drift Between Two Data Sets with Application to UXO Sensing
}

\author{
Xuejun Liao and Lawrence Carin \\ Department of Electrical and Computer Engineering \\ Duke University \\ Durham, NC 27708-0291, USA \\ $\{x j l i a o$, lcarin\}@ee.duke.edu
}

\begin{abstract}
To achieve good generalization in supervised learning, the training and testing examples are usually required to be drawn from the same source distribution. In this paper we propose a method to relax this requirement in the context of logistic regression. Assuming $\mathcal{D}^{p}$ and $\mathcal{D}^{a}$ are two sets of examples drawn from two different distributions $T$ and $A$ (called concepts, borrowing a term from psychology), where $\mathcal{D}^{a}$ are fully labeled and $\mathcal{D}^{p}$ partially labeled, our objective is to complete the labels of $\mathcal{D}^{p}$. We introduce an auxiliary variable $\mu$ for each example in $\mathcal{D}^{a}$ to reflect its mismatch with $\mathcal{D}^{p}$. Under an appropriate constraint the $\mu$ 's are estimated as a byproduct, along with the classifier. We also present an active learning approach for selecting the labeled examples in $\mathcal{D}^{p}$. The proposed algorithm, called migratory logistic regression (MigLogit), is demonstrated successfully on simulated data as well as on real measured data of interest for unexploded ordnance (UXO) cleanup.
\end{abstract}

\section{INTRODUCTION}

In supervised classification problems, the goal is to design a classifier using the training examples (labeled data) such that the classifier predicts the labels correctly for unlabeled test data. The accuracy of the predictions is significantly affected by the quality of the training examples, which are assumed to contain essential information about the test instances for which predictions are desired. A common assumption utilized by learning algorithms is that the training examples and the test instances are drawn from the same source distribution.

As a practical example, consider the detection of a concealed entity based on sensor data collected in a non-invasive manner. This problem is of relevance in several practical problems, for example in the medical imaging of potential tumors or other hidden anomalies. In the context of remote sensing, one is often challenged with the problem of detecting and characterizing a concealed (e.g., underground) target based on remotely collected sensor data. In an example that will be considered explicitly in this paper, consider the detection of buried unexploded ordnance (UXO) [1]. An unexploded ordnance is a bomb that did not explode upon impact with the ground, and such items pose great danger if disturbed (excavated) without care. Sensors used for detecting and characterizing UXO include magnetometers and electromagnetic induction [1]. In designing an algorithm for characterization of anomalies detected by such sensors, to determine if a given buried item is UXO or clutter, one typically requires training data. Such training data typically comes from other former bombing sites that have been cleaned, and there is a significant issue as to whether such extant labeled sensor data are relevant for a new site under test. The challenge addressed in this paper involves learning the relevance and relationship of existing labeled (training) data for analysis of a new unlabeled or partially labeled data set of interest. This type of problem has significant practical relevance for UXO sensing, for which results are presented on measured data, as well as for the aforementioned classes of problems, for which there is uncertainty concerning the appropriateness of existing labeled data for a new set of unlabeled data of interest.

To place this problem in a mathematical setting, let $\mathcal{T}(\mathbf{x}, y)$ be the probability distribution (or concept, borrowing a term from psychology ${ }^{1}$ ) from which test instances (each including a feature vector $\mathbf{x}$ and the associated class label $y$ ) are drawn. The goal in classifier design is to minimize a loss function $L(y, \zeta(\mathbf{x}))$, which is a quantitative measure for the loss incurred by the classifier when it predicts $\zeta(\mathbf{x})$ for $\mathbf{x}$ whose true label is $y$. The minimization is performed for $N$ independent training examples $(\mathbf{x}, y)$ drawn from $\mathcal{T}(\mathbf{x}, y)$, leading to the empirical loss minimization [3], [4]

$$
\min _{\zeta} \frac{1}{N} \sum_{i=1}^{N} L\left(y_{i}, \zeta\left(\mathbf{x}_{i}\right)\right), \quad \text { with } \quad(\mathbf{x}, y) \sim \mathcal{T}(\mathbf{x}, y)
$$

The empirical loss is known to approach the true loss when $N \rightarrow \infty$.

A learning algorithm based on the empirical loss minimization in (1) implicitly assumes that the future test instances are also drawn from $\mathcal{T}(\mathbf{x}, y)$. It is this assumption that assures that the classifier generalizes to test instances when it is trained to minimize empirical loss on training examples. This assumption, however, is often violated in practice, since training examples and test instances may correspond to different collections of measurements (likely performed at different times under different experimental conditions) and the class memberships of the measurements may also change. These issues can introduce statistical differences between the

\footnotetext{
${ }^{1}$ Traditionally, the (probabilistic) mapping $\operatorname{Pr}(y \mid \mathbf{x})$ is called a concept, and $\operatorname{Pr}(\mathbf{x})$ is called a virtual concept (language describing the concept) [2]. For simplicity, usually they are collectively called a concept.
} 
training examples and the test instances; the UXO-sensing problem discussed above constitutes an important example for which the aforementioned statistical issues hold, concerning the utility of existing labeled (training) data.

Assume that one has training examples from a distribution $\mathcal{A}(\mathbf{x}, y)$ which is different from $\mathcal{T}(\mathbf{x}, y)$. For convenience of exposition, we call $\mathcal{T}(\mathbf{x}, y)$ the primary or target distribution and call $\mathcal{A}(\mathbf{x}, y)$ the auxiliary distribution. Accordingly, the examples drawn from $\mathcal{T}(\mathbf{x}, y)$ are called primary data and the examples drawn from $\mathcal{A}(\mathbf{x}, y)$ are called auxiliary data.

In order to write the empirical loss for $\mathcal{T}$ in terms of examples drawn from $\mathcal{A}$, one may employ the technique of importance sampling [5]. By doing so, one makes the following modifications to the expression of empirical loss (1)

$$
\frac{1}{N} \sum_{i=1}^{N} \frac{\mathcal{T}(\mathbf{x}, y)}{\mathcal{A}(\mathbf{x}, y)} L\left(y_{i}, \zeta\left(\mathbf{x}_{i}\right)\right), \quad \text { with } \quad(\mathbf{x}, y) \sim \mathcal{A}(\mathbf{x}, y)
$$

where $\frac{\mathcal{T}(\mathbf{x}, y)}{\mathcal{A}(\mathbf{x}, y)}$ is the importance weight. It is known that the modification does not change the asymptotic behavior provided that $\mathcal{A}(\mathbf{x}, y)$ has the same nonzero support as $\mathcal{T}(\mathbf{x}, y)$.

Unfortunately, both $\mathcal{A}(\mathbf{x}, y)$ and $\mathcal{T}(\mathbf{x}, y)$ are unknown to the algorithm; all that is available are samples from $\mathcal{A}(\mathbf{x}, y)$. The challenge, therefore, is to learn a classifier on training examples drawn from $\mathcal{A}(\mathbf{x}, y)$ such that the resulting classifier still generalizes to test instances drawn from $\mathcal{T}(\mathbf{x}, y)$. Clearly, without assuming any knowledge about the relationship between $\mathcal{A}(\mathbf{x}, y)$ and $\mathcal{T}(\mathbf{x}, y)$, there is little one can do but to treat the training examples as if they are from the target distribution. This will, of course, introduce errors, which may be intolerable when the difference between $\mathcal{A}(\mathrm{x}, y)$ and $\mathcal{T}(\mathbf{x}, y)$ is large.

The problem of learning on examples from one distribution with the goal of generalizing to instances from a different distribution has been addressed in different contexts, using different names. In the following, we provide a brief review of this previous work.

\section{A. Tackling Concept Drifts in Time-Varying Data}

Many data come naturally in streams, collected over a period of time. Such applications include weather recordings, sales and customer data, surveillance video streams, to name a few. For streamed data, it is natural to consider online learning, in which the leaner is dynamically presented with the true label after it makes a prediction and updates its hypothesis based on newly received true labels. When the streamed data are recorded over an extended period of time, the statistics in the data are likely to change. The time-dependent variation of statistics in streamed data are termed concept drift in the literature [6], [2], [7], [8], [9].

Concept drift falls under the general formulation in (2). Here the target distribution characterizes the statistics of the most recent recordings, and there is an auxiliary distribution characterizing the recordings in each time interval in the past. The goal in concept-drift learning is to employ the available recordings to build up the target concept (i.e., the mapping from a feature vector to the associated class label) for the current moment. An important notion is the age of each recording, which determines the utility of the recording to the current prediction.

Three widely used methods for handling concept drift are: time windows [6], instance weighting [10], and ensemble learning [9]. In the first method, a time window is applied to the data stream and the data within the window are employed to build the classifier for the current concept. The window keeps moving towards the future so that the most recent recording is always included in the window. The window acts like a limited memory, with the data outside the window forgotten. A key issue is to determine the window size, which should accurately capture the rate of concept drift. The method of instance weighting is based on the observation that the importance of a past example to the current concept does not change abruptly but rather decreases gradually. A weight is assigned to each past example to reflect its importance. Assuming that concept drift is monotonic (i.e., newer examples are always more important than older ones), one can construct the weights according to the age of each example; for example, one can choose weights that decrease exponentially with the age of examples [10]. The method of ensemble learning maintains an ensemble of classifiers, instead of a single one, for the current concept. This is done by dividing the data stream into chunks and learning a classifier based on each data chunk. The relevance of each classifier to the current concept is evaluated by the generalization error when applying the classifier to the most recent data chunk. The relevance is employed as a weight applied to each classifier and the weighted classifiers are employed to make predictions for the current data chunk.

\section{B. Sample Selection Bias in Econometrics}

In econometrics, the observed data are often a nonrandomly selected sample of the true distribution of interest. If the distribution of interest is $\mathcal{T}$, the selection bias results in samples drawn from $\mathcal{A}$ which is different from $\mathcal{T}$. Heckman [11] developed a method to correct the sample-selection bias for linear regression models. The basic idea of Heckman's method is that if one can estimate the probability of an observation being selected into the sample, one can use this probability estimate to correct the selection bias.

Heckman's model has recently been extended to classification problems [12], where it is assumed that the test instances are drawn from $\mathcal{T}(\mathbf{x}, y)=\operatorname{Pr}(\mathbf{x}, y)$ while the training examples are drawn from $\mathcal{A}(\mathbf{x}, y)=\operatorname{Pr}(\mathbf{x}, y \mid s=1)$, where the variable $s$ controls the selection of training examples: if $s=1$, $(\mathbf{x}, y)$ is selected into the training set; if $s=0,(\mathbf{x}, y)$ is not selected into the training set. Evidently, unless $s$ is independent of $(\mathbf{x}, y), \operatorname{Pr}(\mathbf{x}, y \mid s=1) \neq \operatorname{Pr}(\mathbf{x}, y)$ and hence $\mathcal{T}(\mathbf{x}, y)$ is different from $\mathcal{T}(\mathbf{x}, y)$. By Bayes rule,

$$
\frac{\operatorname{Pr}(\mathbf{x}, y)}{\operatorname{Pr}(\mathbf{x}, y \mid s=1)}=\frac{\operatorname{Pr}(s=1)}{\operatorname{Pr}(s=1 \mid \mathbf{x}, y)}
$$

or

$$
\frac{\mathcal{T}(\mathbf{x}, y)}{\mathcal{A}(\mathbf{x}, y)}=\frac{\operatorname{Pr}(s=1)}{\operatorname{Pr}(s=1 \mid \mathbf{x}, y)}
$$


plugging this into (2), one has

$$
\begin{aligned}
& \frac{1}{N} \sum_{i=1}^{N} \frac{\operatorname{Pr}(s=1)}{\operatorname{Pr}(s=1 \mid \mathbf{x}, y)} L\left(y_{i}, \zeta\left(\mathbf{x}_{i}\right)\right) \\
& \text { with } \quad(\mathbf{x}, y) \sim \operatorname{Pr}(\mathbf{x}, y \mid s=1)
\end{aligned}
$$

which implies that if one has access to $\frac{\operatorname{Pr}(s=1)}{\operatorname{Pr}(s=1 \mid \mathbf{x}, y)}$ one can correct the selection bias by using the empirical loss as expressed in (5). In the special case when $\operatorname{Pr}(s=1 \mid \mathbf{x}, y)=$ $\operatorname{Pr}(s=1 \mid \mathbf{x})$, one may estimate $\operatorname{Pr}(s=1 \mid \mathbf{x})$ from a sufficient sample of $\operatorname{Pr}(\mathbf{x}, s)$ if such a sample is available [12]. In the general case, however, it is difficult to estimate $\frac{\operatorname{Pr}(s=1)}{\operatorname{Pr}(s=1 \mid \mathbf{x}, y)}$, as we do not have a sufficient sample of $\operatorname{Pr}(\mathbf{x}, y, s)$ (if we do, we already have a sufficent sample of $\operatorname{Pr}(\mathbf{x}, y)$, which contradicts the assumption of the problem).

\section{Overview of This Work}

In this paper we propose an efficient algorithm for solving the general problem of learning on examples from $\mathcal{A}$ with the goal of generalizing to instances from $\mathcal{T}$, when $\mathcal{A}$ is different from $\mathcal{T}$. We consider the case in which we have a fully labeled auxiliary data set $\mathcal{D}^{a}$ and a partially labeled primary data set $\mathcal{D}^{p}=\mathcal{D}_{l}^{p} \cup \mathcal{D}_{u}^{p}$, where $\mathcal{D}_{l}^{p}$ are labeled and $\mathcal{D}_{u}^{p}$ unlabeled. We assume that $\mathcal{D}^{p}$ are examples of the primary concept $\mathcal{T}$ (the concept we are interested in) and $\mathcal{D}^{a}$ are examples of the auxiliary concept $\mathcal{A}$ (the one providing indirect and lowquality information about $\mathcal{T}$ ). Our objective is to use a mixed training set $\mathcal{D}^{t r}=\mathcal{D}_{l}^{p} \cup \mathcal{D}^{a}$ to train a classifier that predicts the labels of $\mathcal{D}_{u}^{p}$ accurately, with the hope that $\mathcal{D}_{l}^{p}$ is required to have a small number of examples.

Assume $\mathcal{D}^{p} \sim \operatorname{Pr}(\mathbf{x}, y)$. In light of (3), we can write $\mathcal{D}^{a} \sim \operatorname{Pr}(\mathbf{x}, y \mid s=1)$ as long as the source distributions of $\mathcal{D}^{p}$ and $\mathcal{D}^{a}$ have the same support of nonzero probability ${ }^{2}$. As explained previously, it is difficult to correct the mismatch by directly estimating $\frac{\operatorname{Pr}(s=1)}{\operatorname{Pr}(s=1 \mid \mathbf{x}, y)}$. Therefore we take an alternative approach. We introduce an auxiliary variable $\mu_{i}$ for each $\left(\mathbf{x}_{i}^{a}, y_{i}^{a}\right) \in \mathcal{D}^{a}$ to reflect its mismatch with $\mathcal{D}^{p}$ and to control its participation in the learning process. The $\mu$ 's play a similar role as the weighting factors $\frac{\operatorname{Pr}(s=1)}{\operatorname{Pr}(s=1 \mid \mathbf{x}, y)}$ in (5). However, unlike the weighting factors, the auxiliary variables are estimated along with the classifier in the learning. We employ logistic regression as a specific classifier and develop our method in this context.

The remainder of the paper is organized as follows. A detailed description of the proposed method is provided in Section II, followed by description of a fast learning algorithm in Section III and a theoretical discussion in Section IV. In Section $\mathrm{V}$ we present a method to actively define $\mathcal{D}_{l}^{p}$ when $\mathcal{D}_{l}^{p}$ is initially empty. In Section VI we demonstrate the ideas presented here using simulated data, as well as real data of interest for detecting unexploded ordnance (UXO). Finally, Section VII provides conclusions.

\footnotetext{
${ }^{2}$ For any $\operatorname{Pr}(\mathbf{x}, y \mid s=1) \neq 0$ and $\operatorname{Pr}(\mathbf{x}, y) \neq 0$, there exists $\frac{\operatorname{Pr}(s=1)}{\operatorname{Pr}(s=1 \mid \mathbf{x}, y)}=$ $\frac{\operatorname{Pr}(\mathbf{x}, y)}{\operatorname{Pr}(\mathbf{x}, y \mid s=1)} \in(0, \infty)$ such that (3) is satisfied. For $\operatorname{Pr}(\mathbf{x}, y \mid s=1)=$ $\operatorname{Pr}(\mathbf{x}, y)=0$, any $\frac{\operatorname{Pr}(s=1)}{\operatorname{Pr}(s=1 \mid \mathbf{x}, y)} \neq 0$ makes (3) satisfied.
}

\section{Migratory Logistic Regression (MigLogit): LEARNING JOINTLY ON THE PRIMARY AND AUXILIARY DATA}

We assume $\mathcal{D}_{l}^{p}$ are fixed and nonempty, and without loss of generality, we assume $\mathcal{D}_{l}^{p}$ are always indexed prior to $\mathcal{D}_{u}^{p}$, i.e., $\mathcal{D}_{l}^{p}=\left\{\left(\mathbf{x}_{i}^{p}, y_{i}^{p}\right)\right\}_{i=1}^{N_{l}^{p}}$ and $\mathcal{D}_{u}^{p}=\left\{\left(\mathbf{x}_{i}^{p}, y_{i}^{p}\right)\right.$ : $y_{i}^{p}$ missing $\}_{i=N_{l}^{p}+1}^{N^{p}}$. We use $N^{a}, N^{p}$, and $N_{l}^{p}$ to denote the size (number of data points) in $\mathcal{D}^{a}, \mathcal{D}^{p}$, and $\mathcal{D}_{l}^{p}$, respectively. In Section $\mathrm{V}$ we discuss how to actively determine $\mathcal{D}_{l}^{p}$ when $\mathcal{D}_{l}^{p}$ is initially empty. We consider the binary classification problem and the labels $y^{a}, y^{p} \in\{-1,1\}$. For notational simplicity, we let $\mathbf{x}$ always include a 1 as its first element to accommodate a bias (intercept) term, thus $\mathbf{x}^{p}, \mathbf{x}^{a} \in \mathbb{R}^{d+1}$ where $d$ is the number of features. For a primary data point $\left(\mathbf{x}_{i}^{p}, y_{i}^{p}\right) \in \mathcal{D}_{l}^{p}$, we follow standard logistic regression to write

$$
\operatorname{Pr}\left(y_{i}^{p} \mid \mathbf{x}_{i}^{p} ; \mathbf{w}\right)=\sigma\left(y_{i}^{p} \mathbf{w}^{T} \mathbf{x}_{i}^{p}\right)
$$

where $\mathbf{w} \in \mathbb{R}^{d+1}$ is a column vector of classifier parameters and $\sigma(\eta)=\frac{1}{1+\exp (-\eta)}$ is the sigmoid function. For a auxiliary data point $\left(\mathbf{x}_{i}^{a}, y_{i}^{a}\right) \in \mathcal{D}^{a}$, we define

$$
\operatorname{Pr}\left(y_{i}^{a} \mid \mathbf{x}_{i}^{a} ; \mathbf{w}, \mu_{i}\right)=\sigma\left(y_{i}^{a} \mathbf{w}^{T} \mathbf{x}_{i}^{a}+y_{i}^{a} \mu_{i}\right)
$$

where $\mu_{i}$ is an auxiliary variable. Assuming the examples in $\mathcal{D}_{l}^{p}$ and $\mathcal{D}^{a}$ are drawn i.i.d., we have the log-likelihood function

$$
\begin{aligned}
& \ell\left(\mathbf{w}, \boldsymbol{\mu} ; \mathcal{D}_{l}^{p} \cup \mathcal{D}^{a}\right) \\
& =\sum_{i=1}^{N_{l}^{p}} \ln \sigma\left(y_{i}^{p} \mathbf{w}^{T} \mathbf{x}_{i}^{p}\right)+\sum_{i=1}^{N^{a}} \ln \sigma\left(y_{i}^{a} \mathbf{w}^{T} \mathbf{x}_{i}^{a}+y_{i}^{a} \mu_{i}\right)
\end{aligned}
$$

where $\boldsymbol{\mu}=\left[\mu_{1}, \cdots, \mu_{N^{a}}\right]^{T}$ is a column vector of all auxiliary variables.

The auxiliary variable $\mu_{i}$ is introduced to reflect the mismatch of $\left(\mathbf{x}_{i}^{a}, y_{i}^{a}\right)$ with $\mathcal{D}^{p}$ and to control its participation in the learning of $\mathbf{w}$. A larger $y_{i}^{a} \mu_{i}$ makes $\operatorname{Pr}\left(y_{i}^{a} \mid \mathbf{x}_{i}^{a} ; \mathbf{w}, \mu_{i}\right)$ less sensitive to $\mathbf{w}$. When $y_{i}^{a} \mu_{i}=\infty, \operatorname{Pr}\left(y_{i}^{a} \mid \mathbf{x}_{i}^{a} ; \mathbf{w}, \mu_{i}\right)=1$ becomes completely independent of $\mathbf{w}$. Geometrically, the $\mu_{i}$ is an extra intercept term that is uniquely associated with $\mathbf{x}_{i}^{a}$ and causes it to migrate towards class $y_{i}^{a}$. If $\left(\mathbf{x}_{i}^{a}, y_{i}^{a}\right)$ is mismatched with the primary data $\mathcal{D}^{p}$, w cannot make $\sum_{i=1}^{N_{l}^{p}} \ln \sigma\left(y_{i}^{p} \mathbf{w}^{T} \mathbf{x}_{i}^{p}\right)$ and $\ln \sigma\left(y_{i}^{a} \mathbf{w}^{T} \mathbf{x}_{i}^{a}\right)$ large at the same time. In this case $\mathbf{x}_{i}^{a}$ will be given an appropriate $\mu_{i}$ to allow it to migrate towards class $y_{i}^{a}$, so that $\mathbf{w}$ is less sensitive to $\left(\mathbf{x}_{i}^{a}, y_{i}^{a}\right)$ and can focus more on fitting $\mathcal{D}_{l}^{p}$. Evidently, if the $\mu$ 's are allowed to change freely, their influence will override that of $\mathbf{w}$ in fitting the auxiliary data $\mathcal{D}^{a}$ and then $\mathcal{D}^{a}$ will not participate in learning $\mathbf{w}$. To prevent this from happening, we introduce constraints on $\mu_{i}$ and maximize the log-likelihood subject to the constraints:

$$
\begin{array}{cl}
\max _{\mathbf{w}, \boldsymbol{\mu}} & \ell\left(\mathbf{w}, \boldsymbol{\mu} ; \mathcal{D}_{l}^{p} \cup \mathcal{D}^{a}\right) \\
\text { subject to } & \frac{1}{N^{a}} \sum_{i=1}^{N^{a}} y_{i}^{a} \mu_{i} \leq C, \quad C \geq 0 \\
& y_{i}^{a} \mu_{i} \geq 0, \quad i=1,2, \cdots, N^{a}
\end{array}
$$

where the inequalities in (11) reflect the fact that in order for $\mathbf{x}_{i}^{a}$ to fit $y_{i}^{a}=1$ (or $y_{i}^{a}=-1$ ) we need to have $\mu_{i}>$ 0 (or $\mu_{i}<0$ ), if we want $\mu_{i}$ to exert a positive influence in the fitting process. Under the constraints in (11), a larger 
value of $y_{i}^{a} \mu_{i}$ represents a larger mismatch between $\left(\mathbf{x}_{i}^{a}, y_{i}^{a}\right)$ and $\mathcal{D}^{p}$ and accordingly makes $\left(\mathbf{x}_{i}^{a}, y_{i}^{a}\right)$ play a less important role in determining $\mathbf{w}$. The classifier resulting from solving the problem in (9)-(11) is referred to as migratory logistic regression (MigLogit).

The $C$ in (10) reflects the average mismatch between $\mathcal{D}^{a}$ and $\mathcal{D}^{p}$ and controls the average participation of $\mathcal{D}^{a}$ in determining $\mathbf{w}$. It can be learned from data if we have a reasonable amount of $\mathcal{D}_{l}^{p}$. However, in practice we usually have no or very scarce $\mathcal{D}_{l}^{p}$ to begin with. In this case, we must rely on other information to set $\mathrm{C}$. We will come back to a more detailed discussion on $C$ in Section IV.

\section{FAST LEARNING ALgORITHM}

The optimization problem in (9), (10), and (11) is concave and any standard technique can be utilized to find the global maxima. However, there is a unique $\mu_{i}$ associated with every $\left(\mathbf{x}_{i}^{a}, y_{i}^{a}\right) \in \mathcal{D}^{a}$, and when $\mathcal{D}^{a}$ is large using a standard method to estimate $\mu$ 's can consume most of the computational time.

In this section, we give a fast algorithm for training MigLogit, by taking a block-coordinate ascent approach [13], in which we alternately solve for $\mathbf{w}$ and $\boldsymbol{\mu}$, keeping one fixed when solving for the other. The algorithm draws its efficiency from the analytic solution of $\boldsymbol{\mu}$, which we establish in the following theorem. Proof of the theorem is given in the appendix, and Section IV contains a discussion that helps to understand the theorem from an intuitive perspective.

Theorem 1: Let $f(z)$ be a twice continuously differentiable function and its second derivative $f^{\prime \prime}(z)<0$ for any $z \in \mathbb{R}$. Let $b_{1} \leq b_{2} \leq \cdots \leq b_{N}, R \geq 0$, and

$$
n=\max \left\{m: m b_{m}-\sum_{i=1}^{m} b_{i} \leq R, 1 \leq m \leq N\right\}
$$

Then the problem

$$
\begin{array}{cc}
\max _{\left\{z_{i}\right\}} & \sum_{i=1}^{N} f\left(b_{i}+z_{i}\right) \\
\text { subject to } & \sum_{i=1}^{N} z_{i} \leq R, \quad R \geq 0 \\
& z_{i} \geq 0, \quad i=1,2, \cdots, N
\end{array}
$$

has a unique global solution

$$
z_{i}= \begin{cases}\frac{1}{n} \sum_{j=1}^{n} b_{j}+\frac{1}{n} R-b_{i}, & 1 \leq i \leq n \\ 0, & n<i \leq N\end{cases}
$$

For a fixed $\mathbf{w}$, the problem in (9)-(11) is simplified to maximizing $\sum_{i=1}^{N^{a}} \ln \sigma\left(y_{i}^{a} \mathbf{w}^{T} \mathbf{x}_{i}^{a}+y_{i}^{a} \mu_{i}\right)$ with respect to $\boldsymbol{\mu}$, subject to $\frac{1}{N^{a}} \sum_{i=1}^{N^{a}} y_{i}^{a} \mu_{i} \leq C, C \geq 0$, and $y_{i}^{a} \mu_{i} \geq 0$ for $i=1,2, \cdots, N^{a}$. Clearly $\ln \sigma(z)$ is a twice continuously differentiable function of $z$ and its second derivative $\frac{\partial^{2}}{\partial z^{2}} \ln \sigma(z)=-\sigma(z) \sigma(-z)<0$ for $-\infty<z<\infty$. Thus Theorem 1 applies. We first solve $\left\{y_{i}^{a} \mu_{i}\right\}$ using Theorem 1, then $\left\{\mu_{i}\right\}$ are trivially solved using the fact $y_{i}^{a} \in\{-1,1\}$. Assume $y_{k_{1}}^{a} \mathbf{w}^{T} \mathbf{x}_{k_{1}}^{a} \leq y_{k_{2}}^{a} \mathbf{w}^{T} \mathbf{x}_{k_{2}}^{a} \leq \cdots \leq y_{k_{N^{a}}}^{a} \mathbf{w}^{T} \mathbf{x}_{k_{N^{a}}}^{a}$, where $k_{1}, k_{2}, \cdots, k_{N^{a}}$ is a permutation of $1,2, \cdots, N^{a}$. Then we can write the solution of $\left\{\mu_{i}\right\}$ analytically,

$$
\mu_{k_{i}}=\left\{\begin{array}{cl}
\frac{1}{n} y_{k_{i}}^{a} \sum_{N^{a}=1}^{n} y_{k_{j}}^{a} \mathbf{w}^{T} \mathbf{x}_{k_{j}}^{a} & \\
+\frac{N^{a}}{n} y_{k_{i}}^{a} C-\mathbf{w}^{T} \mathbf{x}_{k_{i}}^{a} & 1 \leq i \leq n \\
0, & n<i \leq N^{a}
\end{array}\right.
$$

TABLE I

Fast Learning Algorithm of Migratory Logistic Regression (MIGLOGIT)

\begin{tabular}{ll}
\hline Input: $\mathcal{D}^{a} \cup \mathcal{D}_{l}^{p}$ and $C$; Output: $\mathbf{w}$ and $\left\{\mu_{i}\right\}_{i=1}^{N^{a}}$ \\
\hline 1. Initialize $\mathbf{w}$ and $\mu_{i}=0$ for $i=1,2, \cdots, N^{a}$. \\
2. Compute the gradient $\nabla_{\mathbf{w}} \ell$ and Hessian matrix $\nabla_{\mathbf{w}}^{2} \ell$. \\
3. Compute the ascent direction $\mathbf{d}=-\left(\nabla_{\mathbf{w}}^{2} \ell\right)^{-1} \nabla_{\mathbf{w}} \ell$. \\
4. Do a linear search for the step-size $\alpha^{*}=\arg \max _{\alpha} \ell(\mathbf{w}+\alpha \mathbf{d})$. \\
5. Update $\mathbf{w}: \mathbf{w} \leftarrow \mathbf{w}+\alpha^{*} \mathbf{d}$. \\
6. Sort $\left\{y_{i}^{a} \mathbf{w}^{T} \mathbf{x}_{i}^{a}\right\}_{i=1}^{N^{a}}$ in ascending order. Assume the result is \\
\\
$y_{k_{1}}^{a} \mathbf{w}^{T} \mathbf{x}_{k_{1}}^{a} \leq y_{k_{2}} \mathbf{w}^{T} \mathbf{x}_{k_{2}}^{a} \leq \cdots \leq y_{k_{N} a}^{a} \mathbf{w}^{T} \mathbf{x}_{k_{N^{a}}}^{a}$, where \\
7. Find the $n$ using (18). \\
8. Update the auxiliary variables $\left\{\mu_{i}\right\}_{i=1}^{N^{a}}$ using (17). \\
9. Check the convergence of $\ell$ : exit and output $\mathbf{w}$ and $\left\{\mu_{i}\right\}_{i=1}^{N^{a}}$ if \\
converged; go back to 2 otherwise. \\
\hline
\end{tabular}

where

$$
\begin{gathered}
n=\max \left\{m: m y_{k_{m}}^{a} \mathbf{w}^{T} \mathbf{x}_{k_{m}}^{a}-\sum_{i=1}^{m} y_{k_{i}}^{a} \mathbf{w}^{T} \mathbf{x}_{k_{i}}^{a} \leq N^{a} C,\right. \\
\left.1 \leq m \leq N^{a}\right\}
\end{gathered}
$$

For a fixed $\boldsymbol{\mu}$, we use the standard gradient-based method [13] to find $\mathbf{w}$. The main procedures of the fast training algorithm for MigLogit are summarized in Table I, where the gradient $\nabla_{\mathbf{w}} \ell$ and the Hessian matrix $\nabla_{\mathbf{w}}^{2} \ell$ are computed from (8).

\section{Auxiliary Variables and Choice of $C$}

Theorem 1 and its constructive proof in the appendix offers some insight into the mechanism of how the mismatch between $\mathcal{D}^{a}$ and $\mathcal{D}^{p}$ is compensated through the auxiliary variables $\left\{\mu_{i}\right\}$. To make the description easier, we think of each data point $\mathbf{x}_{i}^{a} \in \mathcal{D}^{a}$ as getting principal importance $y_{i}^{a} \mathbf{w}^{T} \mathbf{x}_{i}^{a}$ from $\mathbf{w}$ and additional importance $y_{i}^{a} \mu_{i}$ from a given budget totaling $N^{a} C$ ( $C$ represents the average budget for a single $\mathrm{x}^{a}$ ). From the appendix, $N^{a} C$ is distributed among the auxiliary data $\left\{\mathbf{x}_{i}^{a}\right\}$ by a "smallest-first" rule: the smallest $\mathbf{x}_{k_{1}}^{a}$ (that which has the smallest $y_{k_{1}}^{a} \mathbf{w}^{T} \mathbf{x}_{k_{1}}^{a}$ ), gets a portion $y_{k_{1}}^{a} \mu_{k_{1}}$ from $N^{a} C$ first, and when the total importance $y_{k_{1}}^{a} \mathbf{w}^{T} \mathbf{x}_{k_{1}}^{a}+y_{k_{1}}^{a} \mu_{k_{1}}$ reaches the value of the second smallest $\mathrm{x}_{k_{2}}^{a}, N^{a} C$ becomes equally distributed to $\mathbf{x}_{k_{1}}^{a}$ and $\mathbf{x}_{k_{2}}^{a}$ such that their total importances are always equal. Then, when $y_{k_{1}}^{a} \mathbf{w}^{T} \mathbf{x}_{k_{1}}^{a}+y_{k_{1}}^{a} \mu_{k_{1}}=y_{k_{2}}^{a} \mathbf{w}^{T} \mathbf{x}_{k_{2}}^{a}+y_{k_{2}}^{a} \mu_{k_{2}}$ reach the importance of the third smallest, $N^{a} C$ becomes equally distributed to three of them to make them equal. The distribution continues in this way until the budget $N^{a} C$ is used up. The "smallest-first" rule is essentially a result of the concavity of the logarithmic sigmoid function $\ln \sigma(\cdot)$. The goal is to maximize $\sum_{i=1}^{N^{a}} \ln \sigma\left(y_{i}^{a} \mathbf{w}^{T} \mathbf{x}_{i}^{a}+y_{i}^{a} \mu_{i}\right)$. The concavity of $\ln \sigma(\cdot)$ dictates that for any given portion of $N^{a} C$, distributing it to the smallest makes the maximum gain in $\ln \sigma$.

The $C$ is used as a means to compensate for the loss that $\mathcal{D}^{a}$ may suffer from $\mathbf{w}$. The classifier $\mathbf{w}$ is responsible for correctly classifying both $\mathcal{D}^{a}$ and $\mathcal{D}^{p}$. Because $\mathcal{D}^{a}$ and $\mathcal{D}^{p}$ are mismatched, w cannot satisfy both of them: one must suffer if the other is to gain. As $\mathcal{D}^{p}$ is the primary data set, we want $\mathbf{w}$ to classify $\mathcal{D}^{p}$ as accurately as possible. The auxiliary variables are therefore introduced to represent compensations that $\mathcal{D}^{a}$ get from $C$. When $\mathrm{x}^{a}$ gets small contribution from $\mathbf{w}$ and is 
small, it is because $\mathbf{x}^{a}$ is mismatched and in conflict with $\mathcal{D}^{p}$ (assuming perfect separation of $\mathcal{D}^{a}$, no conflict exists among themselves). By the "smallest first" rule, the most mismatched $\mathbf{x}^{a}$ gets compensation first.

A high compensation $y_{i}^{a} \mu_{i}$ whittles down the participation of $\mathbf{x}_{i}^{a}$ in learning $\mathbf{w}$. This is readily seen from the contribution of $\left(\mathbf{x}_{i}^{a}, y_{i}^{a}\right)$ to $\nabla_{\mathbf{w}} \ell$ and $\nabla_{\mathbf{w}}^{2} \ell$, which are obtained from (8) as $\sigma\left(-y_{i}^{a} \mathbf{w}^{T} \mathbf{x}_{i}^{a}-y_{i}^{a} \mu_{i}\right) y_{i}^{a} \mathbf{x}_{i}^{a}$ and $-\sigma\left(-y_{i}^{a} \mathbf{w}^{T} \mathbf{x}_{i}^{a}-\right.$ $\left.y_{i}^{a} \mu_{i}\right) \sigma\left(y_{i}^{a} \mathbf{w}^{T} \mathbf{x}_{i}^{a}+y_{i}^{a} \mu_{i}\right) \mathbf{x}_{i}^{a} \mathbf{x}_{i}^{a T}$, respectively. When $y_{i}^{a} \mu_{i}$ is large, $\sigma\left(-y_{i}^{a} \mathbf{w}^{T} \mathbf{x}_{i}^{a}-y_{i}^{a} \mu_{i}\right)$ is close to zero and hence the contribution of $\left(\mathbf{x}_{i}^{a}, y_{i}^{a}\right)$ to $\nabla_{\mathbf{w}} \ell$ and $\nabla_{\mathbf{w}}^{2} \ell$ are ignorable. We in fact do not need an infinitely large $y_{i}^{a} \mu_{i}$ to make the contributions of $\mathbf{x}_{i}^{a}$ ignorable, because $\sigma(\mu)$ is almost saturated at $\mu= \pm 6$. If $y_{i}^{a} \mathbf{w}^{T} \mathbf{x}_{i}^{a}=-6, \sigma\left(-y_{i}^{a} \mathbf{w}^{T} \mathbf{x}_{i}^{a}\right)=$ 0.9975, implying a large contribution of $\left(\mathbf{x}_{i}^{a}, y_{i}^{a}\right)$ to $\nabla_{\mathbf{w}} \ell$, which happens when $\mathbf{w}$ assigns $\mathbf{x}_{i}^{a}$ to the correct class $y_{i}^{a}$ with probability of $\sigma\left(y_{i}^{a} \mathbf{w}^{T} \mathbf{x}_{i}^{a}\right)=\sigma(-6)=0.0025$ only. In this nearly worst case, a compensation of $y_{i}^{a} \mu_{i}=12$ can effectively remove the contribution of $\left(\mathbf{x}_{i}^{a}, y_{i}^{a}\right)$ because $\sigma\left(-y_{i}^{a} \mathbf{w}^{T} \mathbf{x}_{i}^{a}-y_{i}^{a} \mu_{i}\right)=\sigma(6-12)=\sigma(-6)=0.0025$. To effectively remove the contributions of $N^{m}$ auxiliary data, one needs a total budge $12 N^{m}$, resulting in an average budget $C=12 N^{m} / N^{a}$.

To make a right choice of $C$, the $N^{m} / N^{a}$ should represent the rate that $\mathcal{D}^{a}$ are mismatched with $\mathcal{D}^{p}$. This is because we want $N^{a} C$ to be distributed only to that part of $\mathcal{D}^{a}$ that is mismatched with $\mathcal{D}^{p}$, thus permitting us to use the remaining part in learning w. The quantity $N^{m} / N^{a}$ is usually unknown in practice. However, $C=12 N^{m} / N^{a}$ gives one a sense of at least what range $C$ should be in. As $0 \leq N^{m} \leq N^{a}$, letting $0 \leq C \leq 12$ is usually a reasonable choice. In our experiences, the performance of MigLogit is relatively robust to $C$, as demonstrated in Section VI-B.

\section{Active Selection of $\mathcal{D}_{l}^{p}$}

In Section II we assumed that $\mathcal{D}_{l}^{p}$ had already been determined. In this section we describe how $\mathcal{D}_{l}^{p}$ can be actively selected from $\mathcal{D}^{p}$, based on the Fisher information matrix [14], [15]. The approach is known as active learning [16], [17].

Let $\mathbf{Q}$ denote the Fisher information matrix of $\mathcal{D}_{l}^{p} \cup \mathcal{D}^{a}$ about w. By definition of the Fisher information matrix [18], $\mathbf{Q}=\mathbb{E}_{\left\{y_{i}^{p}\right\},\left\{y_{i}^{a}\right\}} \frac{\partial \ell}{\partial \mathbf{w}} \frac{\partial \ell}{\partial \mathbf{w}}{ }^{T}$, and substituting (8) into this equation gives (a brief derivation is given in the appendix)

$$
\mathbf{Q}=\sum_{i=1}^{N_{l}^{p}} \sigma_{i}^{p}\left(1-\sigma_{i}^{p}\right) \mathbf{x}_{i}^{p} \mathbf{x}_{i}^{p T}+\sum_{i=1}^{N^{a}} \sigma_{i}^{a}\left(1-\sigma_{i}^{a}\right) \mathbf{x}_{i}^{a} \mathbf{x}_{i}^{a T}
$$

where $\sigma_{i}^{p}=\sigma\left(\mathbf{w}^{T} \mathbf{x}_{i}^{p}\right)$ for $i=1,2, \ldots, N_{l}^{p}$, and $\sigma_{i}^{a}=$ $\sigma\left(\mathbf{w}^{T} \mathbf{x}_{i}^{a}+\mu_{i}\right)$ for $i=1,2, \ldots, N^{a}$, and $\mathbf{w}$ and $\left\{\mu_{i}\right\}$ represent the true classifier and auxiliary variables.

It is well known the inverse Fisher information $\mathbf{Q}^{-1}$ lower bounds the covariance matrix of the estimated $w$ [18]. In particular, $[\operatorname{det}(\mathbf{Q})]^{-1}$ lower bounds the product of variances of the elements in $\mathbf{w}$. The goal in selecting $\mathcal{D}_{l}^{p}$ is to reduce the variances, or uncertainty, of $\mathbf{w}$. Thus we seek the $\mathcal{D}_{l}^{p}$ that maximize $\operatorname{det}(\mathbf{Q})$.

The selection proceeds in a sequential manner. Initially $\mathcal{D}_{u}^{p}=\mathcal{D}^{p}, \mathcal{D}_{l}^{p}$ is empty, and $\mathbf{Q}=\sum_{i=1}^{N^{a}} \sigma_{i}^{a}\left(1-\sigma_{i}^{a}\right) \mathbf{x}_{i}^{a} \mathbf{x}_{i}^{a T}$. Then one at a time, a data point $\mathbf{x}_{i}^{p} \in \mathcal{D}_{u}^{p}$ is selected and moved from $\mathcal{D}_{u}^{p}$ to $\mathcal{D}_{l}^{p}$. This causes $\mathbf{Q}$ to be updated as: $\mathbf{Q} \leftarrow \mathbf{Q}+\sigma_{i}^{p}\left(1-\sigma_{i}^{p}\right) \mathbf{x}_{i}^{p}\left(\mathbf{x}_{i}^{p}\right)^{T}$. At each iteration, the selection is based on

$$
\begin{aligned}
& \max _{\mathbf{x}_{i}^{p} \in \mathcal{D}_{u}^{p}} \operatorname{det}\left\{\mathbf{Q}+\sigma_{i}^{p}\left(1-\sigma_{i}^{p}\right) \mathbf{x}_{i}^{p}\left(\mathbf{x}_{i}^{p}\right)^{T}\right\} \\
& =\max _{\mathbf{x}_{i}^{p} \in \mathcal{D}_{u}^{p}}\left\{1+\sigma_{i}^{p}\left(1-\sigma_{i}^{p}\right)\left(\mathbf{x}_{i}^{p}\right)^{T} \mathbf{Q}^{-1} \mathbf{x}_{i}^{p}\right\}
\end{aligned}
$$

where we assume the existence of $\mathbf{Q}^{-1}$, which can often be assured by using sufficient auxiliary data $\mathcal{D}^{a}$.

Evaluation of (20) requires the true values of $\mathbf{w}$ and $\left\{\mu_{i}\right\}$, which are not known a priori. We follow [14] and replace them with the $\mathbf{w}$ and $\left\{\mu_{i}\right\}$ that are estimated from $\mathcal{D}^{a} \cup \mathcal{D}_{l}^{p}$, where $\mathcal{D}_{l}^{p}$ are the primary labeled data selected up to the present.

\section{RESULTS}

In this section the performance of MigLogit is demonstrated and compared to the standard logistic regression. The MigLogit is trained using $\mathcal{D}^{a} \cup \mathcal{D}_{l}^{p}$, where $\mathcal{D}_{l}^{p}$ are either randomly selected from $\mathcal{D}^{p}$, or actively selected from $\mathcal{D}^{p}$ using the method in Section $\mathrm{V}$. When $\mathcal{D}_{l}^{p}$ are randomly selected, 50 independent trials are performed and the results are obtained as an average over the trials. Three logistic regression classifiers are trained using different combinations of $\mathcal{D}^{a}$ and $\mathcal{D}_{l}^{p}: \mathcal{D}^{a} \cup \mathcal{D}_{l}^{p}, \mathcal{D}_{l}^{p}$ alone, and $\mathcal{D}^{a}$ alone, where $\mathcal{D}_{l}^{p}$ are identical to the $\mathcal{D}_{l}^{p}$ used by MigLogit. The four classifiers are tested on $\mathcal{D}_{u}^{p}=\mathcal{D}^{p} \backslash \mathcal{D}_{l}^{p}$ to produce the test-error rate or the area under the ROC curve. Calculation of test error rates is based on the following decision rule: declare $y^{p}=-1$ if $\sigma\left(\mathbf{w}^{T} \mathbf{x}^{p}\right) \leq 0.5$ and $y^{p}=1$ otherwise, for any $\mathbf{x}^{p} \in \mathcal{D}_{u}^{p}$.

The performance of MigLogit is demonstrated on two problem domains. The first is a simulated example and the second is detection of unexploded ordnance (UXO) where the UXO signatures are site-sensitive.

Throughout this section the $C$ in MigLogit is set to $C=$ 6 when the comparison is made to logistic regression. In addition, we present a comparison of MigLogit with different $C$ 's, to examine the sensitivity of MigLogit's performance to C.

\section{A. Synthesized Data}

In the first example, the primary data are simulated as two bivariate Gaussian distributions representing class " -1 " and class " +1 ", respectively. In particular, we have $\operatorname{Pr}\left(\mathbf{x}^{p} \mid y^{p}=\right.$ $-1)=\mathcal{N}\left(\mathbf{x}^{p} ; \boldsymbol{\mu}_{0}, \boldsymbol{\Sigma}\right)$ and $\operatorname{Pr}\left(\mathbf{x}^{p} \mid y^{p}=1\right)=\mathcal{N}\left(\mathbf{x}^{p} ; \boldsymbol{\mu}_{1}, \boldsymbol{\Sigma}\right)$, where the Gaussian parameters $\boldsymbol{\mu}_{0}=[0,0]^{T}, \boldsymbol{\mu}_{1}=[2.3,2.3]^{T}$, and $\boldsymbol{\Sigma}=\left[\begin{array}{cc}1.75 & -0.433 \\ -0.433 & 1.25\end{array}\right]$. The auxiliary data $\mathcal{D}^{a}$ are then a selected draw from the two Gaussian distributions, as described in [12]. We take the selection probability $\operatorname{Pr}\left(s \mid \mathbf{x}^{p}, y^{p}=-1\right)=\sigma\left(w_{0}+w_{1} K\left(\mathbf{x}^{p}, \boldsymbol{\mu}_{0}^{s} ; \boldsymbol{\Sigma}\right)\right)$ and $\operatorname{Pr}\left(s \mid \mathbf{x}^{p}, y^{p}=+1\right)=\sigma\left(w_{0}+w_{1} K\left(\mathbf{x}^{p}, \boldsymbol{\mu}_{1}^{s} ; \boldsymbol{\Sigma}\right)\right)$, where $\sigma$ is the sigmoid function, $w_{0}=-1, w_{1}=\exp (1), K\left(\mathbf{x}^{p}, \boldsymbol{\mu}_{0}^{s} ; \boldsymbol{\Sigma}\right)=$ $\exp \left\{-0.5\left(\mathbf{x}^{p}-\boldsymbol{\mu}_{0}^{s}\right)^{T} \boldsymbol{\Sigma}^{-1}\left(\mathbf{x}^{p}-\boldsymbol{\mu}_{0}^{s}\right)\right\}$ with $\boldsymbol{\mu}_{0}^{s}=[2,1]^{T}$, and $K\left(\mathbf{x}^{p}, \boldsymbol{\mu}_{1}^{s} ; \boldsymbol{\Sigma}\right)=\exp \left\{-0.5\left(\mathbf{x}^{p}-\boldsymbol{\mu}_{1}^{s}\right)^{T} \boldsymbol{\Sigma}^{-1}\left(\mathbf{x}^{p}-\boldsymbol{\mu}_{1}^{s}\right)\right\}$ with $\boldsymbol{\mu}_{1}^{s}=[0,3]^{T}$. We obtain 150 samples of $\mathcal{D}^{p}$ and 150 samples of $\mathcal{D}^{a}$, which are shown in Figure 3.

The MigLogit and logistic regression classifiers are trained and tested as explained at the beginning of this section. The 
results are represented as test error rate as a function of number of primary labeled data used in training, and are shown in Figures 1 and 2. Each curve in Figure 1 is an average over 50 independent trials, with each trial having an independent random selection of $\mathcal{D}_{l}^{p}$. Figure 2 presents the active learning results, with $\mathcal{D}_{l}^{p}$ actively selected as described in Section V.

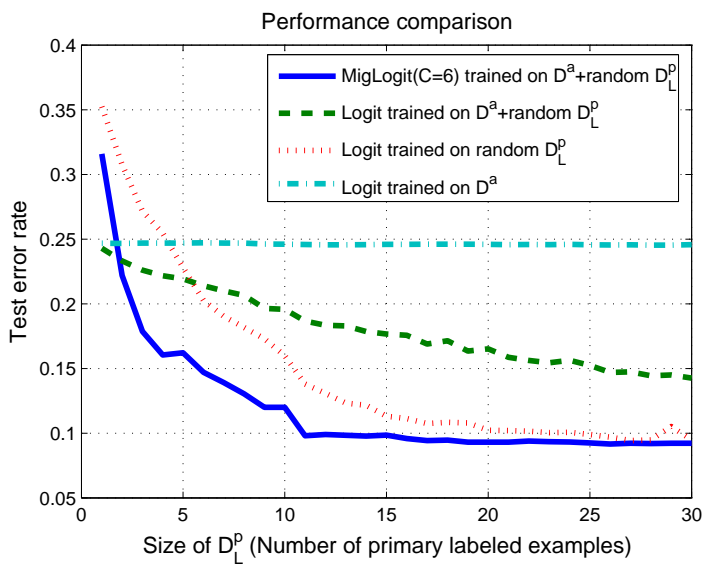

Fig. 1. Test error rates of MigLogit and logistic regression on the synthesized data, as a function of size of $\mathcal{D}_{l}^{p}$. The primary labeled data $\mathcal{D}_{l}^{p}$ are randomly selected from $\mathcal{D}^{p}$. The error rates are an average over 50 independent trials of random selection of $\mathcal{D}_{l}^{p}$.

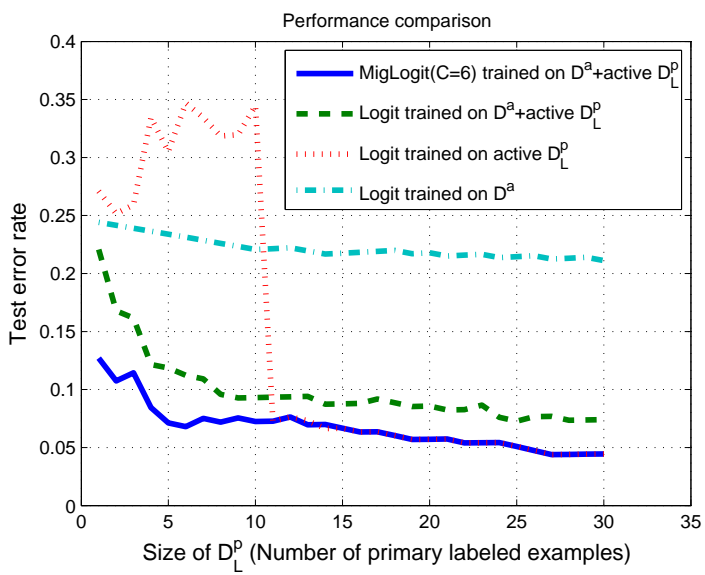

Fig. 2. Error rates of MigLogit and logistic regression on the synthesized data, as a function of size of $\mathcal{D}_{l}^{p}$. The primary labeled data $\mathcal{D}_{l}^{p}$ are actively selected from $\mathcal{D}^{p}$, using the method in Section V.

Several observations are made from inspection of Figures 1 and 2 .

- The MigLogit consistently outperforms the three standard logistic regression classifiers, by a considerable margin. This improvement is attributed to a selective usage of the examples in $\mathcal{D}^{a}$. In particular, each example in $\mathcal{D}^{a}$ is employed according to its agreement with $\mathcal{D}_{l}^{p}$ : a good agreement warrants a higher contribution to determination of the classifier while a poor agreement makes the contribution discounted. The selectivity is implemented through the auxiliary variables which are estimated based on a few examples from $\mathcal{D}^{p}$.
- The performance of the logistic regression trained on $\mathcal{D}_{l}^{p}$ alone changes significantly with the size of $\mathcal{D}_{l}^{p}$. This is understandable, considering that $\mathcal{D}_{l}^{p}$ are the only examples determining the classifier. The abrupt drop of errors from iteration 10 to iteration 11 in Figure 2 may be because the label found at iteration 11 is critical to determining $\mathbf{w}$.

- The logistic regression trained on $\mathcal{D}^{a}$ alone performs significantly worse than MigLogit, reflecting a marked mismatch between $\mathcal{D}^{a}$ and $\mathcal{D}^{p}$.

- The logistic regression trained on $\mathcal{D}^{a} \cup \mathcal{D}_{l}^{p}$ improves, but mildly, as $\mathcal{D}_{l}^{p}$ grows, and it is ultimately outperformed by the the logistic regression trained on $\mathcal{D}_{l}^{p}$ alone, demonstrating that some data in $\mathcal{D}^{a}$ are mismatched with $\mathcal{D}^{p}$ and hence cannot be correctly classified along with $\mathcal{D}^{p}$, if the mismatch is not compensated.

- As $\mathcal{D}_{l}^{p}$ grows, the logistic regression trained on $\mathcal{D}_{l}^{p}$ alone finally approaches to MigLogit, showing that without the interference of $\mathcal{D}^{a}$, a sufficient $\mathcal{D}_{l}^{p}$ can define a correct classifier.

- All four classifiers benefit from the actively selected $\mathcal{D}_{l}^{p}$, and this is consistent with the general observation with active learning [16], [17].

The labeled primary examples $\mathcal{D}_{l}^{p}$ play double roles in the learning process. On the one hand they help to find the correct $\mathbf{w}$, and on the other hand they serve as representative primary labeled data in finding the degree of agreement of each auxiliary example with primary data (i.e., estimating the auxiliary variables). Suppose that it requires $n_{1}$ primary labeled examples to find the auxiliary auxiliary variables for compensating the mismatch between $\mathcal{D}^{a}$ and $\mathcal{D}^{p}$, and that it requires $n_{2}$ labeled primary examples alone (without using auxiliary examples) to find the correct classifier. One may conjecture that $n_{1}$ is smaller than $n_{2}$. Although we have not proven this rigorously, the results in Figures 1 and 2 provide empirical evidence for this being true: note that MigLogit uses much fewer primary labeled examples to find the correct classifier than Logit trained on $\mathcal{D}_{l}^{p}$ does.

The double roles assumed by the primary labeled examples make it a critical issue how to select these examples. We already see that actively selected examples give significant boost to the performance. This makes it clear that active learning is a more appropriate strategy than pure random selection, and contributes in an important manner to the proposed method.

To better understand the active selection process, we show in Figure 3 the first few iterations of active learning. Iteration 0 corresponds to the initially empty $\mathcal{D}_{l}^{p}$, and iterations $1,5,10$, 13 respectively correspond to $1,5,10,13$ data points selected accumulatively from $\mathcal{D}_{u}^{p}$ into $\mathcal{D}_{l}^{p}$. Each time a new data point is selected, the $\mathbf{w}$ is re-trained, yielding the different decision boundaries. As can be seen in Figure 3, the decision boundary does not change much after 10 data are selected, demonstrating convergence.

In Figure 3, each auxiliary data point $\mathbf{x}_{i}^{a} \in \mathcal{D}^{a}$ is symbolically displayed with a size in proportion to $\exp \left(-y_{i}^{a} \mu_{i} / 12\right)$, hence a small symbol of auxiliary data corresponds to large $y_{i}^{a} \mu_{i}$ and hence indicates a discounted contribution of the $i$-th 

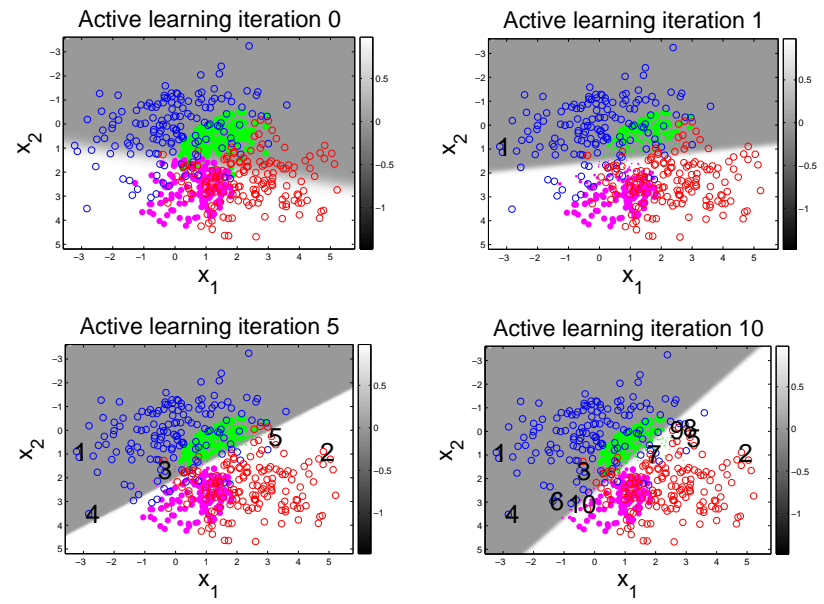

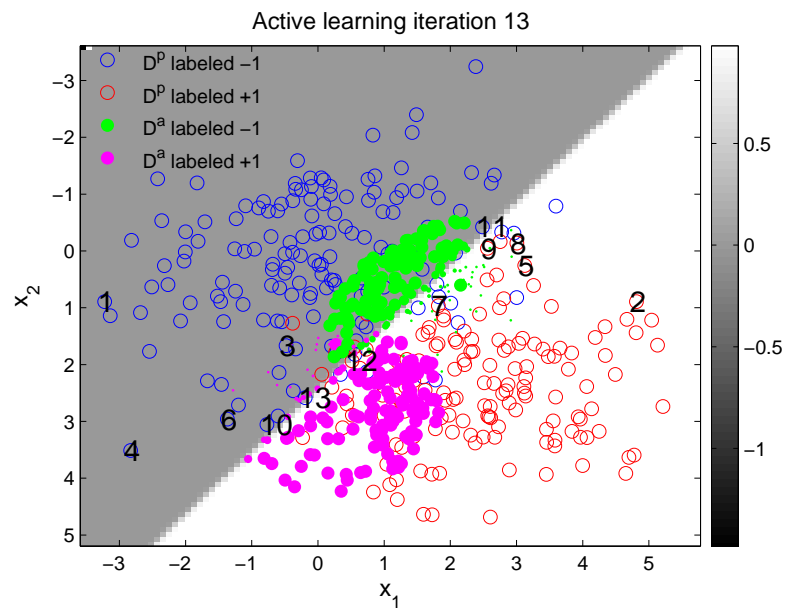

Fig. 3. Illustration of active data selection by MigLogit. Only iterations $0,1,5,10,13$ are shown. The different symbols are defined as: blue $\bigcirc=\mathcal{D}^{p}$ labeled “-1", red $\bigcirc=\mathcal{D}^{p}$ labeled “+1", green $\bullet=\mathcal{D}^{a}$ labeled " -1 ", and magenta $\bullet=\mathcal{D}^{a}$ labeled "+1". The numbers in black denote $\mathcal{D}_{l}^{p}$ and represent the order of selection. The smaller $\bullet$ near the decision boundaries symbolize weakened participation of the associated $\mathcal{D}^{a}$ in determining $\mathbf{w}$. This may only be visible in the zoomed figure (iteration 13).

auxiliary example to determination of $\mathbf{w}$. The auxiliary data that cannot be correctly classified along with the primary data are de-emphasized by the MigLogit. Usually the auxiliary data near the decision boundary are de-emphasized.

\section{B. Application to Detection of Site-Sensitive Unexploded Ord- nance (UXO)}

Unexploded ordnance (UXO) consists of ordnance that did not explode upon impact with the ground. The UXO items are typically buried and consist of significant quantities of metal. Sensing of UXO is typically performed using electromagnetic induction (EMI) and magnetometer sensors. The principal challenge involves distinguishing actual UXO from buried non-ordnance conducting materials. For a more detailed general description of UXO sensing, see [1].

The sensor signature of a given UXO item is dependent on the soil properties as well as the history of the site in which it is located, the latter having a particular strong influence on the signature. The site history is dictated by complex factors such as co-located ordnance, the way the ordnance impacted the soil, and the surrounding man-made conducting clutter and UXO fragments. Therefore UXO detection is a typical sitesensitive problem.

The site-sensitivity makes standard supervised classification techniques an inappropriate choice for UXO detection, due to the difficulty in constituting a universal training set for classifier design. The training examples collected at previous sites are often not appropriate for use for analysis of the current site since the current site is often different from the previous ones (in the sense described above). Despite these disparities, the examples from previous sites are not totally useless; indeed, they can provide quite useful information about the examples for the current site (particularly for the $\mathrm{UXO}$, since the ordnance types at different sites are often the same or similar; the clutter signatures are most often site specific). The usefulness of existing labeled data for a new site of interest is dictated by the characteristics of the new site, as well as on the characteristics of the sites from which the labeled data were acquired; these inter-relationships are complex and often difficult to characterize a priori (often accurate records are not available about the history of a former bombing site).

Let the examples at the current UXO cite be distributed according to $\mathcal{T}(\mathbf{x}, y)$, and the examples at a previous UXO cite be distributed according to $\mathcal{A}(\mathbf{x}, y)$. It is seen that the empirical loss for detection of UXO at the current cite is well described by (2). Therefore one can employ the technique of MigLogit to design the desired classifier.

To demonstrate the utility of MigLogit in UXO detection, we here consider two UXO sites and design the classifier for the primary site (the one we are interested in) by using examples from another site (the auxiliary site). The auxiliary site is called Jefferson Proving Ground (JPG), for which one is provided with the EMI and magnetometer measurements as well the associated labels (which are binary: UXO or non-UXO). The examples from the auxiliary site constitutes the auxiliary data $\mathcal{D}^{a}$. The primary site we are interested in is called Badlands, for which we have unlabeled EMI and magnetometer measurement for constituting the primary data $\mathcal{D}^{p}$. The labeled JPG data consists of 104 total items, of which 16 are UXO and 88 are non-UXO. The Badlands site consists of a total of 492 items, 57 of which are UXO and the remaining 435 are non-UXO. These two former bombing ranges exist at two very different geographical locations within the United States.

The UXO sensor measurements are mapped to four dimensional feature vectors $\left[\log \left(M_{p}\right), \log \left(M_{z}\right), z, \log \left(\frac{M_{p}}{M_{z}}\right)\right]$, where $M_{p}$ and $M_{z}$ are the dipole moments perpendicular and parallel to the target axis, respectively, and $z$ is the approximate target depth [1]. These parameters are estimated by fitting the EMI and magnetometer measurements to a physical model [1]; the features from this study are available upon request to the authors. Each feature is normalized to have zero mean and 


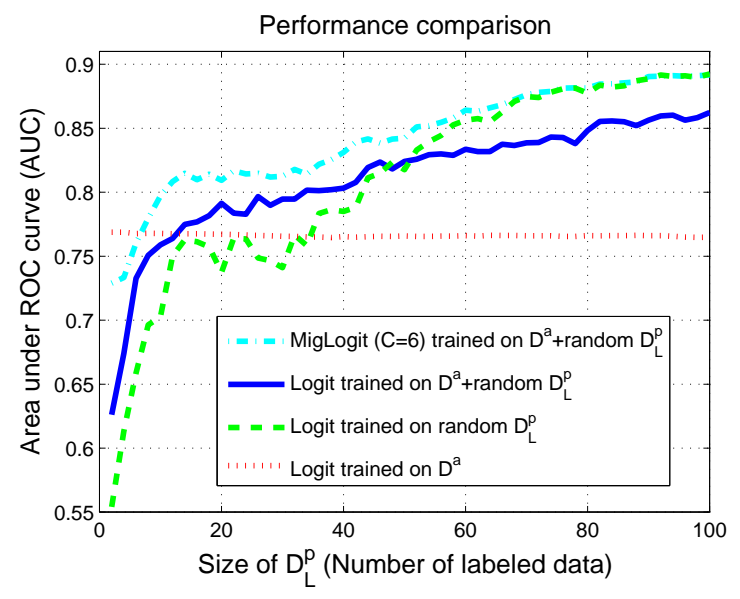

(a) AUC

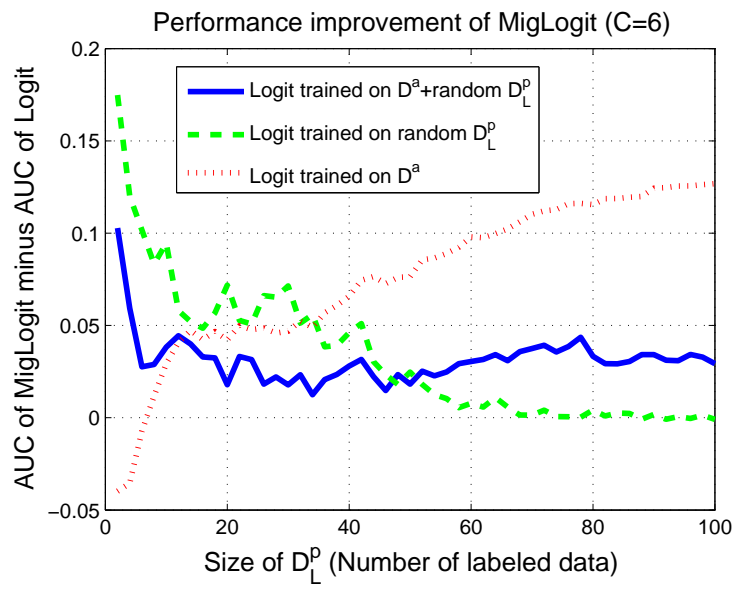

(b) AUC of MigLogit minus AUC of logistic regression

Fig. 4. (a) The area under ROC curve (AUC) of MigLogit and logistic regression on the UXO data, as a function of size of $\mathcal{D}_{l}^{p}$ (b) The AUC of MigLogit minus the AUC's of logistic regression. The auxiliary data are collected at Jefferson Proving Ground (JPG) and the primary data are collected at Badlands. The primary labeled data $\mathcal{D}_{l}^{p}$ are randomly selected from $\mathcal{D}^{p}$. Each curve is an average over 50 independent trials of random selection of $\mathcal{D}_{l}^{p}$.

unitary variance. In UXO detection, one is interested in the receiver's operating characteristic (ROC) curve, particularly the area under ROC curve (AUC) [19].

The results are presented in Figures 4(a) and 5(a), where each curve is the area under ROC curve as a function of the size of $\mathcal{D}_{l}^{p}$. The results in Figure 4(a) are obtained by randomly labeling primary data and by averaging the AUC's over 50 independent trials. The results in Figure 5(a) are obtained by actively labeling primary data using the method in Section V. For a better view of the improvement achieved by MigLogit, we plot in Figures 4(b) and 5(b) the AUC of MigLogit with the AUC of each logistic regression classifier subtracted. A positive difference indicate performance improvement while a negative difference indicates performance degradation. We have the following observations:

- With $\mathcal{D}_{l}^{p}$ determined randomly, MigLogit outperforms all logistic regression classifiers except at the early part of the curves, where there are very few examples in $\mathcal{D}_{l}^{p}$. As

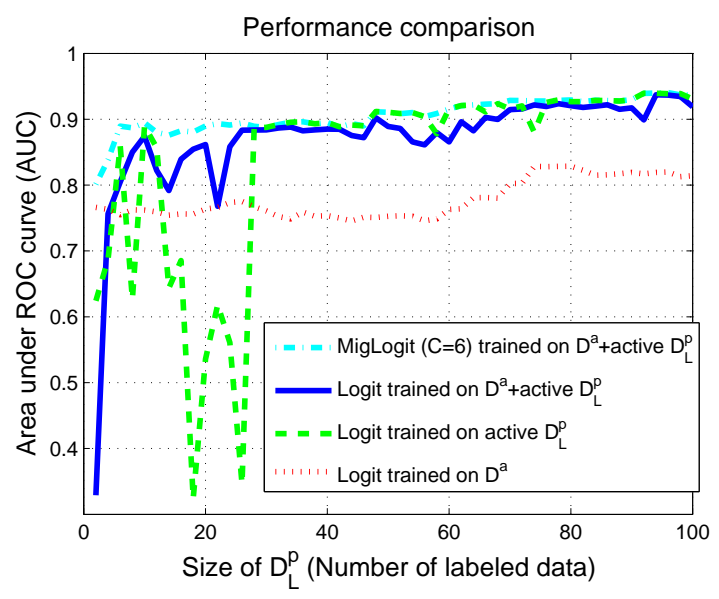

(a) AUC

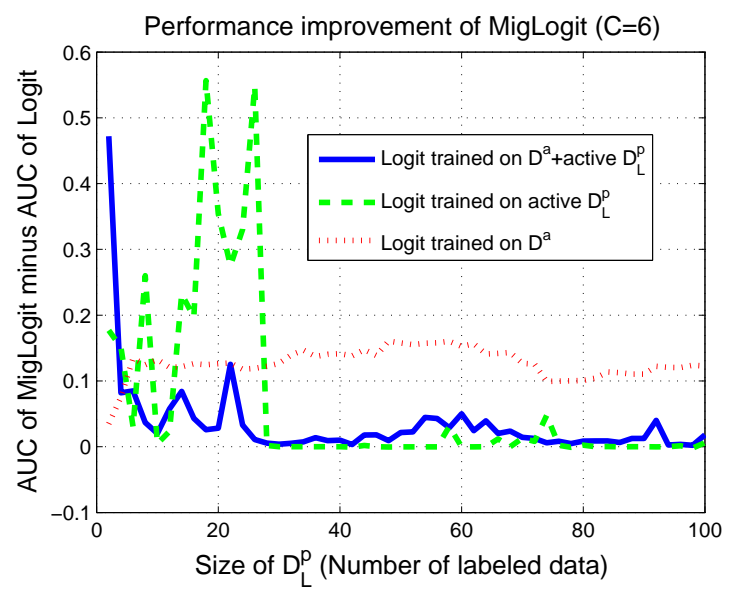

(b) AUC of MigLogit minus AUC of logistic regression

Fig. 5. (a) The area under ROC curve (AUC) of MigLogit and logistic regression on the UXO data, as a function of size of $\mathcal{D}_{l}^{p}$ (b) The AUC of MigLogit minus the AUC's of logistic regression. The auxiliary data are collected at Jefferson Proving Ground (JPG) and the primary data are collected at Badlands. The primary labeled data $\mathcal{D}_{l}^{p}$ are actively selected from $\mathcal{D}^{p}$, based on the method in Section V.

discussed at the end of Section VI-A, the primary labeled examples are critical to the performance of MigLogit. With a few randomly selected examples one may not be able to find the appropriate auxiliary variables, leading to a poor compensation of the mismatch between $\mathcal{D}^{p}$ and $\mathcal{D}^{a}$ and therefore performance degradation.

- With $\mathcal{D}_{l}^{p}$ actively determined, MigLogit outperforms all logistic regression classifiers, regardless of the number of primary labeled examples. This verifies that a good choice of $\mathcal{D}_{l}^{p}$ is important to the performance of MigLogit.

- Active learning is not only beneficial to MigLogit, but to other classifiers as well, again demonstrating the advantage of active learning.

- All conclusions observed in the results on synthesize data extend to the UXO results here.

These observations suggest that MigLogit successfully leverages the auxiliary data from previous UXO sites to quickly find the correct classifier for the new site, requiring much fewer labeled data from the new site than standard 

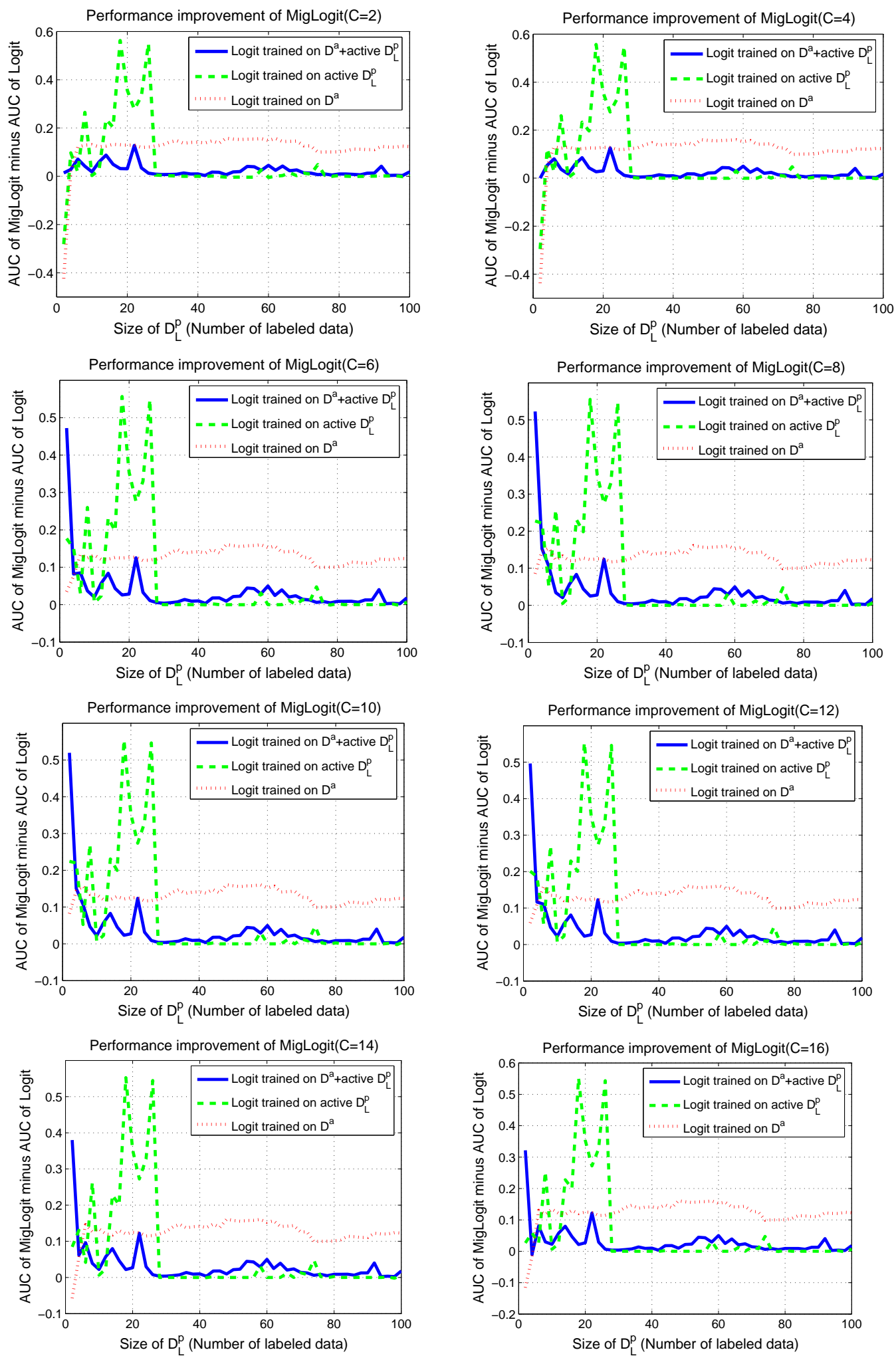

Fig. 6. Performance of MigLogit with different choices of $C$, in the UXO detection problem. The vertical axis is the AUC of MigLogit minus the AUC's of logistic regression. The primary labeled data $\mathcal{D}_{l}^{p}$ are actively selected from $\mathcal{D}^{p}$. From top-left to right-bottom, $C=2,4,6,8,10,12,14,16$.

classifiers. The results for the actual (measured) UXO data suggest that the algorithm captures the concept drift associated with realistic problems of practical importance.

\section{Robustness of MigLogit}

We have discussed in (IV) how to choose $C$ in MigLogit. In this subsection, we show that when the choice is not accurate, 
MigLogit still yields robust results.

We consider the same UXO data and use the same experimental settings as in the previous subsection, except that we vary $C$ in MigLogit to examine its robustness. The $\mathcal{D}_{l}^{p}$ are determined by active learning as described in Section V. We consider eight different values of $C, C=2,4,6,8,10,12,14,16$, to examine the differences in the results obtained under these settings. The results are shown in Figure 6. It is seen that over this wide range of choices for $C$, MigLogit consistently yields superior performances except in a few cases, which occur when the size of $\mathcal{D}_{l}^{p}$ is very small and $C$ is either too large or too small. These results demonstrate the robustness of MigLogit to the choice of $C$, particularly when active learning is invoked. With different $C$, the $\mathcal{D}_{l}^{p}$ are also selected differently, which counteracts the effect of $C$ and increase the robustness of MigLogit.

\section{CONCLUSIONS}

We have proposed an algorithm, called migratory logistic regression (MigLogit), for learning in the presence of concept change between the (auxiliary) training data $\mathcal{D}^{a}$ and the (primary) testing data $\mathcal{D}^{p}$. The basic idea of our method is to introduce an auxiliary variable $\mu_{i}$ for each example $\left(\mathbf{x}_{i}^{a}, y_{i}^{a}\right) \in$ $\mathcal{D}^{a}$, which allows $\mathbf{x}_{i}^{a}$ to migrate to the class $y_{i}^{a}$ when it cannot be correctly classified along with $\mathbf{x}^{p}$ by the classifier. The migrations of $\mathcal{D}^{a}$ are controlled by the inequality constraint $\frac{1}{N^{a}} \sum_{i=1}^{N^{a}} y_{i}^{a} \mu_{i} \leq C$, where $C \geq 0$ is an appropriate bound limiting the average migration. The primary labeled data $\mathcal{D}_{l}^{p}$ play a pivotal role in correctly learning the classifier, and we have presented a method to actively selecting $\mathcal{D}_{l}^{p}$, which enhances the adaptivity of the entire learning process. We have developed a fast learning algorithm to enhance the ability of MigLogit to handle large auxiliary data sets.

The results from both synthesized data and data collected at actual unexploded ordnance (UXO) sites show that MigLogit yields significant improvements over the standard logistic regression, demonstrating that if the classifier trained on $\mathcal{D}^{a}$ is to generalize well to $\mathcal{D}^{p}$, the mismatch between $\mathcal{D}^{a}$ and $\mathcal{D}^{p}$ must be compensated.

In the work presented here it was assumed that we had an existing set of labeled data $\mathcal{D}^{a}$, for which the goal was to learn relationships with a primary set of (unlabeled or partially labeled) data $\mathcal{D}^{p}$. In some problems we may have $M-1$ existing labeled data sets, indexed by $m=1,2, \cdots, M-1$, and we are interested in learning the characteristics (concept) of a new ( $M$-th) unlabeled or partially labeled data set. Using the method presented here, all data in the existing $M-1$ data sets would be combined to define $\mathcal{D}^{a}$. There is a question as to whether this is the most effective way to address this problem. A related technique for handling multiple data sets is based on the notion of multitask learning (MTL) or inductive transfer [20], [21], [22]. Here a task refers to classifier design based on a specific data set. The goal in multitask learning is to enhance training examples used to learn a given task by borrowing information from related tasks. Information borrowing is accomplished by learning the multiple tasks simultaneously under a unified framework. This is particularly beneficial when each task has limited training examples, since information borrowing allows examples of related tasks to be utilized when learning the target task. Note MTL does not require the tasks to be ordered in time; it only assumes that the tasks are related in some manner.

Existing MTL algorithms are distinguished by the way information borrowing is implemented. In a neural network, in which each output node can encode a task [20], [23], [24], information borrowing is implemented by a common internal representations such as hidden nodes and input-tohidden weights. The method in [25] employs a task-kernel to capture the similarity between any two tasks. The taskkernel is used to construct a quadratic regularization term for the parameters across all tasks, which implements information borrowing from one task to another. In Bayesian hierarchical models [26], a common prior distribution is placed over the model parameters in different tasks to represent the information shared between tasks. In nonparametric Bayesian models [27], information borrowing is carried out by by a common Dirichlet process (DP) [28] employed to generate the nonparametric prior distribution over the model parameter in each task. The method in [29] learns a classifier based on a weighting of two tasks, with the auxiliary task given lower weight to reflect that it has a discounted contribution to the classifier learning. Here the target task borrows information from the auxiliary one, through the discounted contribution.

An interesting direction for future research involves examination of the relationship between the concept-drift algorithm presented here and the aforementioned MTL approaches, each of which constitutes a method for implementing transfer learning. In particular, it is of interest to examine the value in retaining the separation of the $M-1$ labeled data sets, as in MTL, versus aggregating them to define $\mathcal{D}^{a}$. For the UXO problem considered as a practical problem in this paper, one may have cleaned $M-1$ previous sites before considering the $M$-th (although this was not the case in the example considered here, in which we only had labeled data from one previous site). This line of investigation will be the focus of a future study.

\section{ACKNOWLEDGMENTS}

The research reported here was supported by the Strategic Environmental Research and Development Program (SERDP).

\section{REFERENCES}

[1] Y. Zhang, L. Collins, H. Yu, C.E. Baum, and L. Carin, "Sensing of unexploded ordnance with magnetometer and induction data: Theory and signal processing," IEEE Trans. Geoscience Remote Sensing, vol. 41, no. 5, pp. 1005-1015, 2003.

[2] G. Widmer and M. Kubat, "Effective learning in dynamic environments by explicit concept tracking," in European Conference on Machine Learning, Pavel B. Brazdil, Ed. 1993, pp. 227-24, SpringerVerlag.

[3] V. Vapnik, Statistical Learning Theory, John Wiley, New York, 1998.

[4] V. N. Vapnik, "An overview of statistical learning theory," IEEE Trans. Neural Networks, vol. 10, no. 5, pp. 988-999, 1999.

[5] C.P. Robert and G. Casella, Monte Carlo Statistical Methods, SpringerVerlag, 1999.

[6] G. Widmer and M. Kubat, "Learning in the presence of concept drift and hidden contexts," Machine Learning, vol. 23, pp. 69-101, 1996.

[7] J. B. Tenenbaum, "Bayesian modeling of human concept learning," in Advances in Neural Information Processing Systems, M. S. Kearns, S. A. Solla, and D. A. Cohn, Eds. 1999, vol. 11, Cambridge, MA: MIT Press. 
[8] R. Klinkenberg and T. Joachims, "Detecting concept drift with support vector machines," in Proceedings of the Seventeenth International Conference on Machine Learning (ICML), P. Langley, Ed., 2000, pp. 487-494.

[9] H. Wang, W. Fan, P. S. Yu, and J. Han, "Mining concept-drifting data streams using ensemble classifiers," in KDD '03: Proceedings of the ninth ACM SIGKDD international conference on Knowledge discovery and data mining, New York, NY, USA, 2003, pp. 226-235, ACM Press.

[10] R. Klinkenberg and S. Ruping, "Concept drift and the importance of examples," in Text Mining - Theoretical Aspects and Applications, J. Franke, G. Nakhaeizadeh, and I. Renz, Eds. 2003, pp. 55-77, PhysicaVerlag, Heidelberg, Germany.

[11] J. Heckman, "Sample selection bias as a specification error," Econometrica, vol. 47, pp. 153-161, 1979.

[12] B. Zadrozny, "Learning and evaluating classifiers under sample selection bias," Proceedings of the 21st ICML, 2004.

[13] D. P. Bertsekas, Nonlinear Programming (2nd Edition), Athena Scientific, 1999.

[14] V. V. Fedorov, Theory of Optimal Experiments, Academic Press, New York, 1972.

[15] D. J. C. MacKay, "Information-based objective functions for active data selection," Neural Computation, vol. 4, pp. 590-604, 1992.

[16] D. A. Cohn, Z. Ghahramani, and M. I. Jordan, "Active learning with statistical models," Advances in Neural Information Processing Systems, vol. 7, pp. 705-712, 1995.

[17] A. Krogh and J. Vedelsby, "Neural network ensembles, cross validation, and active learning," Advances in Neural Information Processing Systems, vol. 7, pp. 231-238, 1995.

[18] T.M. Cover and J.A. Thomas, Elements of Information Theory, John Wiley, New York, 1991.

[19] J. Hanley and B. McNeil, "The meaning and use of the area under a receiver operating characteristic (ROC) curve," Radiology, vol. 143, pp. 29-36, 1982.

[20] R. Caruana, "Multitask learning," Machine Learning, vol. 28, pp. 41-75, 1997.

[21] J. Baxter, "A model of inductive bias learning," Journal of Artificial Intelligence Research, vol. 12, pp. 149-198, 2000.

[22] K. Yu, V. Tresp, and A. Schwaighofer, "Learning gaussian processes from multiple tasks," in The 22nd International Conference on Machine Learning (ICML), 2005.

[23] B. Bakker and T. Heskes, "Task clustering and gating for Bayesian multitask learning," Journal of Machine Learning Research, pp. 83-99, 2003.

[24] X. Liao and L. Carin, "Radial basis function network for multi-task learning," in Advances in Neural Information Processing Systems 18, pp. 795-802. 2006.

[25] T. Evgeniou, C. A. Micchelli, and M. Pontil, "Learning multiple tasks with kernel methods," Journal of Machine Learning Research, pp. 615637, 2005.

[26] F. Dominici, G. Parmigiani, K.H. Reckhow, and R.L. Wolpert, "Combining information from related regressions," Journal of Agricultural, Biological, and Environmental Statistics, vol. 2, no. 3, pp. 313-332, 1997.

[27] Y. Xue, X. Liao, L. Carin, and B. Krishnapuram, "Multi-task learning for classification with dirichlet process priors," Journal of Machine Learning Research (JMLR), vol. 8, pp. 35-63, 2007.

[28] T. Ferguson, "A Bayesian analysis of some nonparametric problems," Annals of Statistics, vol. 1, pp. 209-230, 1973.

[29] P. Wu and T. G. Dietterich, "Improving svm accuracy by training on auxiliary data sources," Proceedings of the 21st ICML, 2004.

\section{APPENDIX}

Proof of Theorem 1: Let $f^{\prime}(z)$ be the first derivative of $f(z)$. We have $\sum_{i=1}^{N} f\left(b_{i}+z_{i}\right)=\sum_{i=1}^{N} f\left(b_{i}\right)+$ $\sum_{i=1}^{N} \int_{0}^{z_{i}} f^{\prime}\left(x+b_{i}\right) d x$. The first term on the right side is a constant and hence, the problem in (13) is equivalent to

$$
\max _{\left\{z_{i}\right\}} \sum_{i=1}^{N} \int_{0}^{z_{i}} f^{\prime}\left(b_{i}+x\right) d x
$$

Because $f^{\prime \prime}(z)<0$, we have for any $\tau_{1} \leq \tau_{2}$ that $f^{\prime}\left(\tau_{1}+x\right) \geq$ $f^{\prime}\left(\tau_{2}+x\right)$ and consequently

$$
\begin{gathered}
\int_{0}^{\Delta} f^{\prime}\left(\tau_{1}+x\right) d x \geq \int_{0}^{\Delta} f^{\prime}\left(\tau_{2}+x\right) d x \\
\forall \quad \tau_{1} \leq \tau_{2} \quad \text { and } \quad \Delta \geq 0
\end{gathered}
$$

By (12), there exists $0 \leq r<n\left(b_{n+1}-b_{n}\right)$ such that $R=n b_{n}-\sum_{k=1}^{n} b_{k}+r=\sum_{k=1}^{n} k \Delta_{k}$ where $\Delta_{k}=b_{k+1}-b_{k}$ for $k=1, \cdots, n-1$, and $\Delta_{n}=r / n$. We now use (A2) to distribute $\Delta_{1}, 2 \Delta_{2}, \cdots, n \Delta_{n}$ to $z_{1}, z_{2}, \cdots, z_{N}$ such that the resulting $\left\{z_{i}\right\}$ maximize (A-1). As $\Delta_{k} \geq 0$ for $k=1, \cdots, n$, and any distribution of $\left\{k \Delta_{k}\right\}_{k=1}^{N}$ to $\left\{z_{k}\right\}_{k=1}^{N}$ makes $\sum_{i=1}^{N} z_{i}=\sum_{k=1}^{n} k \Delta_{k}=R$, the constraints of (14) and (15) are automatically satisfied.

Initially $z_{i}=0$ for $i=1,2, \cdots, N$.

As $\Delta_{1}=b_{2}-b_{1} \geq 0$, by (A-2), $\int_{0}^{\Delta_{1}} f^{\prime}\left(b_{1}+x\right) d x \geq$ $\int_{0}^{\Delta_{1}} f^{\prime}\left(b_{2}+x\right) d x$, therefore $\Delta_{1}$ is distributed to $z_{1}$, i.e., $z_{1} \leftarrow$ $z_{1}+\Delta_{1}$, which makes $b_{1}+z_{1}=b_{2}$.

Similarly $\Delta_{2}=b_{3}-b_{2} \geq 0$, by (A-2), $\int_{0}^{\Delta_{2}} f^{\prime}\left(b_{2}+x\right) d x \geq$ $\int_{0}^{\Delta_{2}} f^{\prime}\left(b_{3}+x\right) d x$, therefore $2 \Delta_{2}$ is equally distributed to $z_{1}$ and $z_{2}$, i.e., $z_{1} \leftarrow z_{1}+\Delta_{2}$ and $z_{2} \leftarrow z_{2}+\Delta_{2}$, which makes $b_{1}+z_{1}=b_{2}+z_{2}=b_{3}$.

Generally, $k \Delta_{k}$ is equally distributed to $z_{1}, z_{2}, \cdots, z_{k}$. After the distribution of $k \Delta_{k}, k=1,2, \cdots, n$, we have $z_{k}=\sum_{i=k}^{n} \Delta_{i}$ for $k=1,2, \cdots, n$ and $z_{k}=0$ for $k=$ $n+1, n+2, \cdots, N$, which is equal to the solution in (16). Because the problem is strictly concave, the solution is unique and globally optimal.

Derivation of Equation (19): By definition of logistic regression, $\mathbf{w}$ is the parameter of the conditional distribution $\operatorname{Pr}(y \mid \mathbf{x})=\sigma\left(y \mathbf{w}^{T} \mathbf{x}\right)$, with $\mathbf{x}$ given and fixed. Let $\mathbf{g}=\partial \ln \sigma\left(y \mathbf{w}^{T} \mathbf{x}\right) / \partial \mathbf{w}=\left[1-\sigma\left(y \mathbf{w}^{T} \mathbf{x}\right)\right] y \mathbf{x}$. Then $\mathbb{E}_{y}\left(\mathbf{g g}^{T}\right)=\sum_{y=-1,1} \sigma\left(y \mathbf{w}^{T} \mathbf{x}\right)\left[1-\sigma\left(y \mathbf{w}^{T} \mathbf{x}\right)\right]^{2} \mathbf{x} \mathbf{x}^{T}$. Using $\sigma\left(-\mathbf{w}^{T} \mathbf{x}\right)=1-\sigma\left(\mathbf{w}^{T} \mathbf{x}\right)$, we obtain $\mathbb{E}_{y}\left(\mathbf{g g}^{T}\right)=\sigma\left(\mathbf{w}^{T} \mathbf{x}\right)[1-$ $\left.\sigma\left(\mathbf{w}^{T} \mathbf{x}\right)\right] \mathbf{x x}^{T}$. Summing $\mathbb{E}\left(\mathbf{g g}^{T}\right)$ over all primary and auxiliary data points (assuming the data are independent), we obtain the formula of $\mathbf{Q}$. 